\title{
Schizophrenia: Neural Mechanisms for Novel Therapies
}

\author{
AKIRA SAWA ${ }^{1,2}$ AND SOLOMON H SNYDER ${ }^{1,2,3}$
}

\begin{abstract}
Although valuable antischizophrenic drugs exist, they only partially ameliorate symptoms and elicit substantial side effects. Classic neuroleptic drugs act by blocking dopamine receptors. They can relieve some symptoms but not behavioral withdrawal features that are designated "negative" symptoms. Clozapine and related newer atypical neuroleptics may be more efficacious in relieving negative symptoms. Understandng their actions may facilitate new drug discovery. Agents influencing glutamate neurotransmission and N-methyl-D-aspartate receptors, especially the cotransmitter D-serine, are promising. Stimulation of the $\alpha 7$ subtype of nicotinic acetylcholine receptor may also be efficacious. The search for genes linked to schizophrenia has revealed several leads that may permit development of novel therapeutic agents. Promising genes include disrupted-in-schizophrenia-1, dysbindin, and neuregulin.
\end{abstract}

\section{INTRODUCTION}

Schizophrenia is a complex disease, or more likely, it is a group of related illnesses with a strong genetic predisposition (1). The biological underpinnings of schizophrenia are evident from multiple studies showing reproducibly enlarged brain ventricles and other abnormalities. This implies defects in brain structures, especially in the temporal and frontal cerebral cortex $(2,3)$. The absence of inflammatory alterations in schizophrenic brains suggests that the defects reflect abnormalities in development $(2,4)$. Gene mapping studies have tracked susceptibility to schizophrenia to a few loci (5). Unfortunately, we still do not know the specific molecular cause of any type of schizophrenia. While the development of therapeutic agents has thus been largely empirical, many highly effective drugs have emerged over the past fifty years. Learning how these drugs act has advanced neurotransmitter research. The treatment of depression and anxiety also has been marked by serendipitous discoveries of drugs leading to novel molecular insights. Indeed, therapeutic agents in psychiatry have been the most powerful tools available for elucidating the roles of neurotransmitters in behavior.

Because neurotransmitters have provided the only route to drug development in schizophrenia thus far, they will occupy much of this review. Since links of beneficial and adverse effects of drugs relate to their initial identification as therapeutic agents, we will emphasize historical features of drug development. We also will show how recent insights into molecular and physiological abnormalities in schizophrenics and disease-selective genetic loci and candidate susceptibility genes may facilitate novel drug discovery.

\section{NEUROTRANSMITTERS}

The brain utilizes at least 50 distinct neurotransmitters, which can be grouped in chemical classes: biogenic amines, amino acids, peptides, and gases. Virtually all drugs in psychiatry act through only a few of these transmitters, generally the biogenic amines: norepinephrine, dopamine, and serotonin; and the amino acid: $\gamma$ aminobutyric acid (GABA). The importance of amines for psychotropic drug action relates in part to their concentration in the limbic areas of the brain, which regulate emotional behavior. Additionally, amine transmitters have been well characterized for more than 50 years, providing maximal opportunity for the pharmaceutical industry to systemically develop agents that influence their biosynthesis, degradation, release, reuptake, and receptor activities. The more recently characterized amino acids and gaseous transmitters participate in pathways that are relevant to psychiatry. In coming years, we should anticipate novel drugs acting through these systems.

\section{Dopamine and Serotonin}

The most important advance in the treatment of schizophrenia occurred 50 years ago with the introduction of chlorpromazine (Table 1). Chlorpromazine was synthesized as an antihistamine but was excessively sedating. The French neurosurgeon Henri Laborit examined it as a preanesthetic medication and found that it elicited a beatific quietude and recommended it to 2 psychiatrists. Jean Delay and Pierre Deniker found it to be highly effective, first in mania and then in schizophrenia. Studies of chlorpromazine and its descendents reveal selective beneficial effects in uniquely schizophrenic symptoms rather than nonspecific sedation. Delay and Deniker made a crucial observation that, with escalating doses, side effects resembling Parkinson's disease appeared in addition to antipsychotic behavior. They coined the term "neuroleptic," meaning "to clasp the neuron," to indicate that the antipsychotic behavior was linked to some sort of neurological influence.

Neuroleptics were linked to dopamine after doses of reserpine resulted in similar antipsychotic behavior. Reserpine depletes the brain of serotonin and dopamine whereas chlorpromazine does not affect levels of these neurotransmitters. Arvid Carlsson (6) discovered selective alterations in metabolites of dopamine

${ }^{1}$ Department of Neuroscience; ${ }^{2}$ Department of Psychiatry and Behavioral Sciences; ${ }^{3}$ Department of Pharmacology and Molecular Sciences, Johns Hopkins University, School of Medicine, Baltimore, MD 21205. 
Table 1. Historic overview of drugs for schizophrenia

\begin{tabular}{|c|c|c|c|c|}
\hline Era & Classification & $\begin{array}{l}\text { Representative } \\
\text { compounds }\end{array}$ & Mechanisms & Efficacy \\
\hline 1950s & Typical neuroleptics & Chlorpromazine (6-8) & D2R blockade & Effective for positive symptoms \\
\hline 1990s & Atypical neuroleptics & Clozapine (9-11) & $\begin{array}{l}\text { Blockade of multiple } \\
\text { receptors }\end{array}$ & $\begin{array}{l}\text { Effective both for positive and some negative } \\
\text { symptoms including D2R and } 5 \mathrm{HT} 2 \mathrm{R} \text {; less EPS }\end{array}$ \\
\hline 1995 & $\begin{array}{l}\text { New atypical } \\
\text { neuroleptics }\end{array}$ & $\begin{array}{l}\text { Olanzepine, Risperidone, } \\
\text { Quetiapine }\end{array}$ & Similar to clozapine & Similar to clozapine \\
\hline \multirow[t]{3}{*}{ Future } & $\begin{array}{l}\text { NMDA-R coagonist } \\
\text { (at glycine site) }\end{array}$ & $\begin{array}{l}\text { D-serine, D-cycloserine, } \\
\text { glycine }(14,15)\end{array}$ & NMDA-R activation & Effective for some negative symptoms \\
\hline & mGluR2/3 agonist & LY354740, LY379268 (19) & $\begin{array}{l}\text { mGluR2/3 activation, } \\
\text { block Glu release }\end{array}$ & $\begin{array}{l}\text { Effective for behavioral abnormalities induced } \\
\text { by PCP in animals }\end{array}$ \\
\hline & Selective $\alpha 7$ agonist & DMXB-A (21) & $\begin{array}{l}\alpha 7 \text { nicotinic receptor } \\
\text { activation }\end{array}$ & Effective for auditory gating deficit \\
\hline
\end{tabular}

after reserpine had been taken, suggesting increased release of dopamine, presumably from augmented firing of dopamine neurons. Carlsson (6) speculated that neuroleptics block dopamine receptors leading to a feedback enhancement of neuronal firing. In this way, neuroleptics and reserpine both would diminish the synaptic activity of dopamine despite an increase in its release that was provoked by neuroleptics. More than a decade after Carlsson's hypothesis, the ability to measure neurotransmitter receptors by ligand binding provided direct demonstration that the relative clinical potencies of neuroleptics closely paralleled their blockade of a subtype of dopamine receptor, the D-2 receptor $(7,8)$. By blocking D-2 receptors in the limbic system of structures that regulate emotions, neuroleptics provide antipsychotic efficacy. Receptor blockade in the caudate and putamen, brain regions that regulate motor behavior, elicits extrapyramidal, Parkinson-like side effects. This type of "typical" neuroleptic has been widely used given its efficacy against "positive" symptoms including delusions, hallucinations, and the peculiar schizophrenic thought disorder. Nonetheless, typical neuroleptics are ineffective against "negative" symptoms, which include affective flattening and social withdrawal.

A role for serotonin in neuroleptic action was suggested by the emergence of clozapine as a therapeutic agent (9-11). This drug was introduced in the 1960s but was withdrawn in several countries because of its ability to induce agranulocytosis. Its unique benefits prompted reintroduction, with the precaution of routine blood monitoring. Clozapine is effective for psychotic symptoms in schizophrenics that display resistance to treatment with typical neuroleptics (11). Even more interesting, it alleviates certain negative symptoms, including loss of capacity to experience pleasure and loss of motivation (11). Clozapine blocks dopamine D-2 receptors at therapeutic doses and probably relieves positive symptoms via mechanisms similar to the typical neuroleptics. How it acts upon negative symptoms is less clear. Clozapine blocks the 5-HT2 subtype of serotonin receptor with substantially greater potency than its influence on D-2 sites. Additionally, it potently blocks $\alpha$-adrenergic, muscarinic cholinergic, and histamine $\mathrm{H}-1$ receptors. Many investigators feel the combination of blocking 5-HT2 and D-2 receptors is key to clozapine's unique therapeutic profile, though actions at other receptors known or unknown, may be equally or more important.

As no one knows exactly how clozapine acts, pharmaceutical companies developed agents mimicking its receptor profile, especially its potent ability to block receptors at 5-HT2 sites. The resulting 2nd generation of "atypical" antipsychotic drugs includes risperidone, olanzapine, quetiapine, and ziprasidone. Like clozapine, these drugs appear to relieve negative and positive symptoms. Unlike clozapine, they do not elicit an unacceptably high incidence of agranulocytosis. The success of the atypical neuroleptics is prompting efforts to definitively establish the mechanisms that cause the beneficial actions of clozapine. As of this writing there are no certain answers.

\section{Glutamate}

A role for glutamate in schizophrenia stems from the drug psychosis approach to the disease. In this model, if a drug elicits a psychosis that faithfully mimics schizophrenia, then elucidating how the drug acts should lead to novel therapeutic approaches. LSD psychosis was advanced as a model for schizophrenia, but most investigators concluded that it did not faithfully mimic its major symptoms. Amphetamine, which facilitates dopamine release, causes a paranoid psychosis that often is indistinguishable from acute paranoid schizophrenia. This fact, coupled with the link of dopamine receptors and neuroleptics, led to a "dopamine hypothesis" of schizophrenia, though no reproducible abnormalities in dopamine disposition have been reported in schizophrenics.

Phencyclidine (PCP) produces a psychotic state that mimics schizophrenia better than any of the other drug-induced psychoses (12). Ketamine, a PCP derivative, also evokes schizophrenic symptoms in normal subjects (13). The discovery that PCP blocks the $N$-methyl-D-aspartate (NMDA) subtype of glutamate receptor suggested a role for these sites in schizophrenia.

The NMDA receptor is unique in possessing distinct recognition sites for 2 neurotransmitters, glutamate, and glycine. Recent evidence suggests that D-serine, the "abnormal" isomer, is the physiologic ligand for the glycine site in most parts of the brain (14). D-serine is generated in protoplasmic astrocytes that ensheathe the synapse by serine racemase and converts L- to D-serine. Several controlled clinical trials have reported therapeutic 
responses to glycine or D-serine when administered in conjunction with classic neuroleptics (15). The responses include improvement in negative symptoms. D-cycloserine, an anti-tuberculosis drug that is a partial agonist at the glycine site, is effective also.

Subtle interactions between GABA- and glutamate-containing neurons may clarify mechanisms of PCP psychosis and facilitate antipsychotic drug development (Figure 1). GABA neurons, which contain NMDA receptors, synapse upon glutamate neurons, which possess on their nerve terminals Group II metabotropic glutamate receptors (mGluR2/3). When glutamate stimulates these mGluR sites, release of glutamate is inhibited. Firing of GABA neurons inhibits firing of glutamate neurons, consistent with GABA's actions as an inhibitory transmitter. By blocking NMDA receptors on GABA neurons, PCP diminishes GABA release. The lessened inhibition of glutamate neurons leads to augmented glutamate release, which is well established in the prefrontal cerebral cortex following PCP treatment (16). Thus, PCP can stimulate glutamate release even though it blocks a subtype of glutamate receptor. The GABA synthesizing enzyme, glutamate decarboxylase, is reportedly diminished in a schizophrenic's prefrontal cortex $(17,18)$. This lack presumably triggers a decrease in GABA release and an augmented glutamate release, similar to treatment of PCP. In the prefrontal cerebral cortex, mGluR2/3 agonist drugs inhibit the increased glutamate release provoked by PCP (19). They also block behavioral abnormalities induced by PCP in animals. Accordingly, mGluR2/3 agonists offer promise as a novel approach to schizophrenic therapy.

\section{Acetylcholine}

Like many other advances in psychiatry, a role for acetylcholinerelated drugs in schizophrenia emerged from astute clinical observation $(20,21)$. Schizophrenics smoke much more than other psychiatric patients, a behavior which is not readily explained by efforts of patients to reduce the sedating effects of their neuroleptic medication. Nicotine transiently relieves some schizophrenic symptoms that are not improved with typical neuroleptics. Nicotinic cholinergic receptors appear to mediate a form of sensory processing which is disturbed in schizophrenics. It has long been known that schizophrenics are unable to screen out extraneous sensory information. This process can be monitored by EEG responses to repeated auditory stimuli. A positive EEG deflection $50 \mathrm{~ms}$ after delivery of auditory stimuli is normally inhibited at the 2nd identical stimulus (P50 auditory gating) (22). This inhibition is markedly lessened in schizophrenics. Abnormalities in auditory gating occur with notable frequency in 1st-degree relatives of schizophrenics. Nicotine gum normalizes the deficient auditory gating in schizophrenic patients, as well as their 1stdegree relatives. Auditory gating can be monitored in mice and is associated with the $\alpha 7$ subtype of nicotinic cholinergic receptors. Mouse strains with the most abnormal auditory gating have the fewest $\alpha 7$ receptors displayed. Agonists at $\alpha 7$ receptors normalize the deficient auditory gating in rodents (21). Interestingly, $\alpha 7$ agonists lack the cardiovascular actions and addictive properties of nicotine. As such, agonists acting at the level of the $\alpha 7$ receptors are under development for clinical trial in schizophrenia.

The $\alpha 7$ receptors may participate directly in the pathophysiology of schizophrenic disturbance. Leonard et al (23) recently
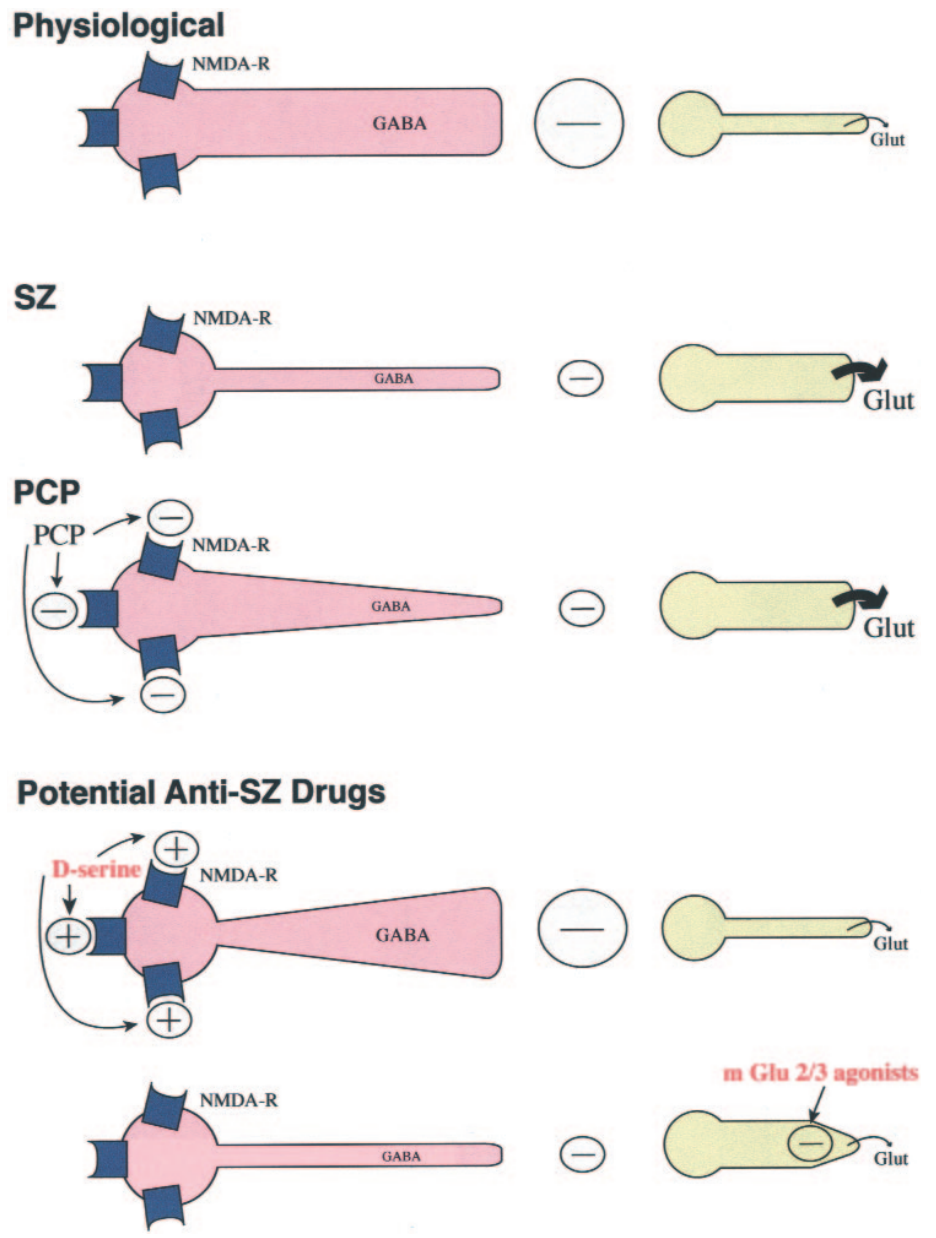

Figure 1. Schematic representation of drug actions that normalize aberrant glutamate neurotransmission in the prefrontal cortex of schizophrenics. GABA neurons (pink) are depicted as synapsing upon glutamate neurons (yellow). In schizophrenics (SZ), the GABA inhibitory neurons in the prefrontal cortex may be decreased in number or functionally impaired, fitting with reports of lowered levels of the GABA-synthesizing enzyme, glutamate decarboxylase, in schizophrenic brains $(17,18)$. PCP diminishes glutamate stimulation of the GABA neurons by blocking the NMDA subtype of glutamate receptor (NMDA-R). The GABA inhibitory neurons synapse upon glutamate neurons so that PCP causes enhanced glutamate efflux in the prefrontal cortex. D-serine is a coagonist with glutamate of NMDA-R at the so-called glycine site so that its administration enhances functions of the GABA inhibitory neurons. Agonists of the mGlu2/3 receptors block excess glutamate efflux, which occurs with PCP administration and which has been speculated to take place in schizophrenia.

reported a higher prevalence of functional promoter mutations in $\alpha 7$ nicotinic acetylcholine receptors (CHRNA7) in schizophrenic subjects than in control subjects; 1 of the mutations may influence the P50 auditory evoked potential response $(22,23)$.

\section{CANDIDATE GENES}

Improvements on existing drugs and drugs acting at novel neurotransmitters will certainly yield improved therapy. However, this empirical approach to therapy is not as intellectually satisfying as efforts to find causes. Genetic loci conferring susceptibility to schizophrenia occur on several chromosomes, and investigators 
Table 2. Candidate susceptibility genes for schizophrenia

\begin{tabular}{lclll} 
Gene & Chromosome & \multicolumn{1}{c}{ Possible influence on neurotransmission } & Reference \\
\hline RGS4 & $1 q 21$ & Modulates G-protein signaling downstream of receptors for dopamine and other neurotransmitters & (40-42) \\
DISC-1 & $1 q 42$ & Interacts with citron, which possibly links to PSD95/glutamate (NMDA) receptor & (34,35,37) \\
Dysbindin & $6 p 24$ & Influences glutamatergic neurotransmission via PSD signaling and clustering \\
Neuregulin 1 & $8 p 21$ & Decreases NMDA-R expression and modulates glutamatergic neurotransmission \\
G72 & $13 q 34$ & Modulates DAAO, influencing D-serine metabolism & (39) \\
CHRNA7 & $15 p 14$ & Acetylcholine receptor & (33) \\
COMT & $22 q 11$ & Influences metabolism of catecholamines such as dopamine \\
PRODH & $22 q 11$ & Functions as a neuromodulator for glutamatergic neurotransmission and serves as precursor for & (29-31) \\
& & glutamate &
\end{tabular}

RGS4, regulator of G-protein signaling-4; PSD, postsynaptic density; CHRNA7, $\alpha 7$ nicotinic acetylcholine receptor; PRODH, proline dehydrogenase.

have tried to narrow down the responsible regions to identify the causal genes for susceptibility (Table 2). Will identification of the "schizophrenia" gene(s) impact therapy? In the best case scenario, an abnormal gene might cause excess formation of a neurotoxin so that a synthetic antitoxin would be therapeutic. However, genetic solutions to schizophrenia may not readily emerge. Huntington's disease, a Mendelian dominant condition much more accessible to analysis than schizophrenia, is caused by a specific mutation in the gene for the protein Huntingtin. While Huntington's disease involves selective brain damage in the caudateputamen and cerebral cortex, Huntingtin is expressed throughout the body. Moreover, we still do not know how the mutated protein selectively damages specific regions of the brain.

The search for genetic loci in schizophrenia has led to several sites related to neurotransmitters that may plausibly be associated with the disease. Other sites are associated with cytoskeletal proteins that influence neuronal outgrowth and might mediate developmental abnormalities that have been implicated in schizophrenic brain. Varying levels of gene expression can be evaluated by microarray analysis, which has revealed alterations in schizophrenic brain for several synaptic marker proteins.

\section{Genes Linked to Putative Neurotransmitters}

Catechol-O-methyltransferase. One locus affected in some schizophrenic families and associated with neurotransmitter disposition occurs at chromosome 22q11. Adults with deletion of 22q11, designated velo-cardio-facial syndrome, DiGeorge syndrome, or 22 deletion syndrome, display an extremely high incidence of schizophrenia, about $25 \%$ to $30 \%$, and 22 q11 deletion occurs in $2 \%$ of diagnosed schizophrenics (24-26). The gene that elicits the symptoms of 22q11 deletion is not known, but there are interesting candidates. For instance, catechol- $O$-methyltransferase (COMT), whose gene is located on $22 q 11$, is 1 of the 2 principal enzymes degrading catecholamines including dopamine. The catecholamines are inactivated either by reuptake into the neurons that released them or through metabolism by monoamine oxidase or COMT. In most areas of the brain, reuptake inactivation predominates so that COMT does not markedly influence catecholamine levels. By contrast, in the prefrontal cerebral cortex, which mediates the cognitive functions that are impaired in schizophrenia, dopamine levels are sensitive to COMT levels. One of the two alleles of the COMT gene that is associated with aug- mented COMT activity also is related to poor performance on certain tests of working memory and to inefficient brain activation as monitored by functional magnetic resonance imaging (27). This allele occurs more often in schizophrenics than in age-matched controls. These studies suggest that a relative deficiency in dopamine transmission in prefrontal cortex may mediate some schizophrenic symptomatology. By contrast, studies of COMT gene knockout mice indicate that depletion of COMT with presumably augmented dopamine transmission, can cause behavioral disturbance (28). Female COMT knockout mice display impaired emotional reactivity in models of anxiety behavior. Of course, one cannot readily equate anxiety behavior in mice with human schizophrenic disturbance. These findings suggest that drugs that influence COMT might be therapeutic in mental illnesses including schizophrenia.

Proline Dehydrogenase. Proline dehydrogenase whose gene is localized, like COMT, to 22q11, also may participate in susceptibility for schizophrenia $(29,30)$. Proline dehydrogenase introduces a double bond into the pyrrolidine ring, setting the stage for cleavage of the ring to glutamate $\gamma$-semialdehyde, which is then readily transformed to glutamate. Jacquet et al (29) reported that a family with 2 schizophrenic subjects had a deletion of $350 \mathrm{~kb}$ within the 22q11 region, which involves the entire locus for the proline dehydrogenase gene. They also identified 2 rare missense mutations associated with high plasma proline levels in 3 schizophrenic patients. In parallel, Liu et al (30) reported genetic variations at the locus for the proline dehydrogenase gene associated with schizophrenia. Support for a behavioral role of proline dehydrogenase comes from studies of proline dehydrogenase knockout mice (31). These animals display a deficit in sensorimotor gating, such as prepulse inhibition, that is characteristic of schizophrenic patients. Besides serving as a precursor of glutamate, proline itself may be a neuromodulater of glutamatergic transmission in the brain. A brain-specific high-affinity proline transporter is expressed selectively in a subset of glutamatergic synapses (32). Endogenous extracellular proline also potentiates excitatory transmission at certain glutamate synapses.

D-serine. D-amino acids may link a deficit of glutamatergic neurotransmission with candidate genes for schizophrenia. Chromosome $13 q$ includes a well-characterized susceptibility locus for 
schizophrenia (5). A systematic single-point association and haplotype analysis with replication in 2 independent populations identified a novel gene at 13q34, called G72, which encodes a protein of 153 amino acid (33). Yeast 2-hybrid analysis shows that the G72 protein interacts with D-amino acid oxidase (DAAO). Under physiological conditions, this enzyme selectively degrades D-serine, the glial neuromodulator of NMDA-glutamate receptors (14). Though the relationships may seem far-fetched, it is conceivable that an abnormality in the 13q34 locus involving G72 influences the metabolism by DAAO of D-serine and thereby alters glutamate neurotransmission. In support of this notion, G72 has been found to activate DAAO several fold (33).

\section{Cytoskeletal Genes}

Disrupted-in-schizophrenia-1. The Disrupted-in-schizophrenia (DISC)-1 gene was discovered in studies of a Scottish family with a chromosomal translocation (34). In the family, $47 \%$ of the family members with the chromosomal abnormality manifested major psychiatric illnesses such as schizophrenia. All the family members with the chromosomal abnormality, including those without notable psychiatric symptoms, displayed diminished levels of certain types of brain potentials, referred to as P300, which are often decreased in schizophrenic patients (35). Disruption of DISC-1 has been reported thus far in only 1 family with schizophrenia. Nonetheless, elucidation of how the mutated protein gives rise to psychosis may shed light on the larger population of schizophrenics. Analogously, rare, genetically determined forms of Alzheimer's disease involve selected genes whose proteins participate in the biosynthesis of the amyloid beta peptides (36). As the peptides are deposited in the senile plaques of all Alzheimer's disease patients, these rare forms of the disease have strongly supported efforts to develop drugs that inhibit the biosynthesis of the peptide. To this end, we have recently characterized DISC-1 protein and its functions (37). The DISC-1 protein consists of 854 amino acids. The translocation that causes the disease involves the deletion of the C-terminal 257 amino acids (34). DISC-1 is developmentally regulated with highest levels in late embryonic development of rats at the time of maximal cerebral cortical neuronal migration (37). DISC-1 is localized in particulate fractions and has structural characteristics resembling a cytoskeletal protein. Yeast 2-hybrid analysis reveals interactions of DISC-1 with several cytoskeletal proteins. Detailed analysis of 1 of these, NUDEL, suggests a role of DISC-1 in neuronal migration and neurite outgrowth. Expression of mutant DISC-1, which fails to bind to NUDEL, leads to altered neuronal outgrowth in PC12 cells. DISC-1 also associates with Citron, a cytoskeletal protein localized to neuronal dendrites in association with the postsynaptic density protein PSD95. As PSD95 is an anchoring protein for the NMDA receptor, DISC-1, through interactions with Citron, might influence NMDA neurotransmission.

Dysbindin. Dysbindin is another cytoskeletal protein that is associated with schizophrenia (38). Linkage analysis had indicated the importance of 6p24-21 for schizophrenia and related disorders (5). A family-based association analysis of polymorphism markers within these regions suggested that single-nucleotide polymorphisms within the gene DTNBP1, the human ortholog of mouse dysbindin, were strongly associated with schizophrenia. Dysbindin was first identified as a protein that binds to $\gamma$-dystrobrevin, a component of the dystrophin protein complex, which is disordered in muscular dystrophy. The dystrophin complex also participates in synaptic systems of the brain, especially cytoskeletal elements in postsynaptic densities. The dystrophin complex regulates the clustering of nicotinic receptors and recruits neuronal nitric oxide synthase to PSD95-facilitating interactions with NMDA receptors. Thus, dysbindin abnormalities might influence glutamate neurotransmission.

Neuregulin 1. Neuregulin 1 is yet another cytoskeletal, synaptic protein which may influence susceptibility to schizophrenia (39). Fine mapping of the $8 \mathrm{p}$ locus and haplotype-association analysis, supplemented by a transmission/disequilibrium test, have identified Neuregulin 1 as a candidate gene for schizophrenia. Neuregulin 1 is expressed at central nervous system synapses and influences the expression and activation of neurotransmitter receptors, including glutamate receptors. Neuregulin 1 regulates development; knockout mice display severe developmental abnormalities in both the nervous system and the heart. Mutant mice heterozygous for Neuregulin 1 display behavioral abnormalities that also are present in mice heterozygous for the Neuregulin 1 receptor, ErbB4. Interestingly, the behavioral abnormalities of the mutant Neuregulin 1 animals are partially reversed by the antipsychotic drug clozapine. Also, the Neuregulin 1 mutant mice express fewer functional NMDA receptors than control mice.

\section{Gene Expression Evaluated by Microarray Analysis}

DNA microarray analysis permits the screening of thousands of genes in disease states. Such approaches have revealed selectively decreased expression in schizophrenic brains of genes associated with presynaptic functions such as $N$-ethylmaleimide sensitive factor (NSF) and synapsin II (40). These proteins might provide clues to the pathophysiology of the disease while also serving as markers to facilitate genetic and therapeutic investigations. A combined strategy employing microarray analysis, association and linkage analyses implicated the regulator of G-protein signalling-4 (RGS4) as a susceptibility gene for schizophrenia $(41,42)$. The RGS family of about 20 proteins is a class of GTPase activating proteins (GAPs) that augment the degradation of GTP bound to G-proteins and hence shortens the duration of G-proteinrelated synaptic signaling. All the major biogenic amines and amino acid neurotransmitters, to varying extents, signal via G-protein coupled receptors. Microarray analysis revealed a decrease in schizophrenic prefrontal cortex of RGS4 but not of 10 other RGS family members. Moreover, 274 other genes associated with G-protein signaling were unchanged. The alterations were not likely caused by treatment with the neuroleptic haloperidol, used by many of the patients, because monkeys chronically treated with haloperidol failed to show such alterations. RGS4 maps to locus 1q 21-22, a chromosome region which is strongly linked to schizophrenia (43).

Abnormalities in several physiological and biochemical markers in schizophrenics and their close relatives may facilitate the tailoring of medication to specific individuals. For instance, 
EEG recordings have identified an event-related potential designated P300, which is reproducibly diminished in schizophrenics (44). The P300 abnormality behaves very much like a genetic marker as in the case of the DISC-1 Scottish family described above (34). In instances where individuals with reduced P300 are not overtly schizophrenic, researchers have speculated that such individuals are likely to develop schizophrenia and might warrant prophylactic therapy. Such markers are referred to as "intermediate phenotypes" (45) and include P50, P300, saccadic eye movement, and performance in the Wisconsin Card Sort Test.

While therapies based on genetic abnormalities and other markers may be distant, better drugs based on neurotransmitters continue to offer considerable short-term promise. Though bonafide schizophrenia has an incidence of about $1 \%$, far larger numbers of individuals may be part of a "schizophrenia spectrum", apparently sane but emotionally withdrawn and manifesting social maladjustment. Such individuals might benefit from treatment with antipsychotic agents that elicit fewer side effects. A similar situation has revolutionized treatment of mild depression. The traditional tricyclic antidepressants caused troublesome side effects such as dry mouth, difficulty urinating, weight gain, and sexual dysfunction. The new generation of antidepressants, exemplified by fluoxetine (Prozac), have fewer side effects and have greatly altered our appreciation of the "depression spectrum" of illnesses. It had been thought that tricyclic antidepressants only benefit patients with serious biologic depression. The benign sideeffect profile of fluoxetine and related agents, such as paroxetine (Paxil) and sertraline (Zoloft), permitted widespread use with dramatic therapeutic efficacy in many patients whose depression had been thought too mild to warrant drug treatment.

With the wide diversity of genetic, neurotransmitter, physiological, and clinical strategies being employed, we anticipate important advances in treating schizophrenia, the most disabling of mental illnesses.

\section{ACKNOWLEDGMENTS}

We thank Ms D Dodson for typing the manuscript and Ms $Y$ Lema for arranging the figure. This work is supported by USPHS Grants DA-00266, $\mathrm{MH}-18501$, and Research Scientist Award DA-00074 (SHS), and grants from the Stanley Medical Research Institute, NARSAD, and S-R foundation (Washington DC, USA) (AS).

Address correspondence and reprint requests to Solomon H Snyder, Department of Neuroscience, Johns Hopkins University, School of Medicine, 725 N. Wolfe Street, Baltimore, MD 21205. Fax: 410-9553623; e-mail: ssnyder@bs.jhmi.edu.

Submitted December 2, 2002; accepted for publication December 23, 2002.

\section{REFERENCES}

1. Sawa A, Snyder SH. (2002) Schizophrenia: diverse approaches to a complex disease. Science 296:692-5.

2. Harrison PJ. (1999) The neuropathology of schizophrenia. A critical review of the data and their interpretation. Brain 122:593-624.

3. Weinberger DR, Torrey EF, Neophytides AN, Wyatt RJ. (1979) Lateral cerebral ventricular enlargement in chronic schizophrenia. Arch. Gen. Psychiatry 36: 735-9.
4. Weinberger DR. (1996) On the plausibility of "the neurodevelopmental hypothesis" of schizophrenia. Neuropsychopharmacology 14:1S-11S.

5. Berrettini WH. (2000) Are schizophrenic and bipolar disorders related? A review of family and molecular studies. Biol. Psychiatry 48:531-8.

6. Carlsson A. (1988) The current status of the dopamine hypothesis of schizophrenia. Neuropsychopharmacology 1:179-86.

7. Creese I, Burt DR, Snyder SH. (1976) Dopamine receptor binding predicts clinical and pharmacological potencies of antischizophrenic drugs. Science 192:481-3.

8. Seeman P, Lee T, Chau-Wong M, Wong K. (1976) Antipsychotic drug doses and neuroleptic/dopamine receptors. Nature 261:717-9.

9. Meltzer HY. (2002) Mechanism of action of atypical antipsychotic drugs. In: Neuropsychopharmacology: the fifth generation of progress. Davis KL, Charney D, Coyle JT, Nemeroff C. (eds.) Raven Press, New York, pp. 819-31.

10. Kapur S, Remington G. (2001) Atypical antipsychotics: new directions and new challenges in the treatment of schizophrenia. Annu. Rev. Med. 52:503-17.

11. Thaker GK, Carpenter Jr WT. (2001) Advance in schizophrenia. Nat. Med. 7:667-71.

12. Javitt DC, Zukin SR. (1991) Recent advances in the phencyclidine model of schizophrenia. Am. J. Psychiatry 148:1301-8.

13. Krystal JH et al. (1994) Subanesthetic effects of the noncompetitive NMDA antagonist, ketamine, in humans. Psychotomimetic, perceptual, cognitive, and neuroendocrine responses. Arch. Gen. Psychiatry 51:199-214.

14. Baranano DE, Ferris CD, Snyder SH. (2001) Atypical neural messengers. Trends. Neurosci. 24:99-106

15. Goff DC, Coyle JT. (2001) The emerging role of glutamate in the pathophysiology and treatment of schizophrenia. Am. J. Psychiatry 158:1367-77.

16. Moghaddam B, Adams BW. (1998) Reversal of phencyclidine effects by a group II metabotropic glutamate receptor agonist in rats. Science 281:1349-52.

17. Akbarian $S$ et al. (1995) Gene expression for glutamic acid decarboxylase is reduced without loss of neurons in prefrontal cortex of schizophrenics. Arch. Gen. Psychiatry 52:258-66.

18. Guidotti A et al. (2000) Decrease in reelin and glutamic acid decarboxylase67 (GAD67) expression in schizophrenia and bipolar disorder: a postmortem brain study. Arch. Gen. Psychiatry 57:1061-69.

19. Schoepp DD, Marek JG. (2002) Preclinical pharmacology of mGlu2/3 receptor agonists: novel agents for schizophrenia? Curr. Drug Targets CNS Neurologic Disorders 1:215-25.

20. Lyon ER. (1999) A review of the effects of nicotine on schizophrenia and antipsychotic medications. Psychiatr. Serv. 50:1346-50.

21. Simosky JK, Stevens KE, and Freedman R. (2002) Nicotinic agonists and psychosis. Curr. Drug Targets CNS Neurologic. Disorders 1:149-62.

22. Light GA, Braff DL. (1998) The "incredible shrinking" P50 event-related potential. Biol. Psychiatry 43:918-20.

23. Leonard $S$ et al. (2002) Association of promoter variants in the $\alpha 7$ nicotinic acetylcholine receptor subunit gene with an inhibitory deficit found in schizophrenia. Arch. Gen. Psychiatry 59:1085-96.

24. Bassett AS, Chow EW. (1999) 22q 11 deletion syndrome: a genetic subtype of schizophrenia. Biol. Psychiatry 46:882-91

25. Murphy KC, Jones LA, Owen MJ. (1999) High rates of schizophrenia in adults with velo-cardio-facial syndrome. Arch. Gen. Psychiatry 56:940-5.

26. Murphy KC. (2002) Schizophrenia and velo-cardio-facial syndrome. Lance 359:426-30.

27. Egan MF et al. (2001) Effect of COMT Val108/158 Met genotype on frontal lobe function and risk for schizophrenia. Proc. Natl. Acad. Sci. U.S.A. 98:6917-22.

28. Gogos JA et al. (1998) Catechol-O-methyltransferase-deficient mice exhibit sexually dimorphic changes in catecholamine levels and behavior. Proc. Natl. Acad. Sci. U.S.A. 95:9991-6.

29. Jacquet $\mathrm{H}$ et al. (2002) PRODH mutations and hyperprolinemia in a subset of schizophrenic patients. Hum. Mol. Genet. 11:2243-49.

30. Liu H et al. (2002) Genetic variation at the 22q 11 PRODH2/DGCR6 locus presents an unusual pattern and increases susceptibility to schizophrenia. Proc. Natl. Acad. Sci. U.S.A. 99:3717-22.

31. Gogos JA et al. (1999) The gene encoding proline dehydrogenase modulates sensorimotor gating in mice. Nat. Genet. 21:434-9.

32. Renick SE et al. (1999) The mammalian brain high-affinity L-proline transporter is enriched preferentially in synaptic vesicles in a subpopulation of excitatory nerve terminals in rat forebrain. J. Neurosci. 19:21-33.

33. Chumakov I et al. (2002) Genetic and physiological data implicating the new human gene G72 and the gene for D-amino acid oxidase in schizophrenia. Proc. Natl. Acad. Sci. U.S.A. 99:13675-80.

34. Millar JK et al. (2000) Disruption of two novel genes by a translocation cosegregating with schizophrenia. Hum. Mol. Genet. 9:1415-23.

35. Blackwood DH et al. (2001) Schizophrenia and affective disorders-cosegregation with a translocation at chromosome lq42 that directly disrupts brain-expressed genes: clinical and P300 findings in a family. Am. J. Hum. Genet. 69:428-33.

36. Price DL, Sisodia SS. (1998) Mutant genes in familial Alzheimer's disease and transgenic models. Annu. Rev. Neurosci. 21:479-505.

37. Ozeki Y et al. (2003) Disrupted-In-Schizophrenia-1 (DISC-1): Mutant truncation prevents binding to NUDEL and inhabits neurite outgrowth. Proc. Natl. Acad. Sci. U.S.A. 100:289-94.

38. Straub RE et al. (2002) Genetic variation in the 6p22.3 gene DTNBP1, the human ortholog of the mouse dysbindin gene, is associated with schizophrenia. Am. J. Hum. Genet. 71:337-48.

39. Stefansson $\mathrm{H}$ et al. (2002) Neuregulin 1 and susceptibility to schizophrenia Am. J. Hum. Genet. 71:877-92.

40. Mirnics K, Middleton FA, Marquez A, Lewis DA, Levitt P. (2000) Molecular char- 
acterization of schizophrenia viewed by microarray analysis of gene expression in prefrontal cortex. Neuron 28:53-67.

41. Mirnics K, Middleton FA, Stanwood GD, Lewis DA, Levitt P. (2001) Diseasespecific changes in regulator of G-protein signaling 4 (RGS4) expression in schizophrenia. Mol. Psychiatry 6:293-301.

42. Chowdari KV et al. (2002) Association and linkage analyses of RGS4 polymorphisms in schizophrenia. Hum. Mol. Genet. 11:1373-80.
43. Brzustowicz LM, Hodgkinson KA, Chow EW, Honer WG, Bassett AS. (2000) Location of a major susceptibility locus for familial schizophrenia on chromosome 1q21-q22. Science 288:678-82.

44. Ford JM. (1999) Schizophrenia: the broken P300 and beyond. Psychophysiology 36:667-82.

45. Moldin SO. (1994) Indicators of liability to schizophrenia: perspectives from genetic epidemiology. Schizophr. Bull. 20:169-84 